\title{
Green Function Method for Nonlinear Systems
}

\author{
Marco Frasca* \\ Via Erasmo Gattamelata, 3 \\ 00176 Roma (Italy) \\ (Dated: February 7, 2008)
}

\begin{abstract}
We show that a Green function solution can be given for a class of non-homogeneous nonlinear systems having relevance in quantum field theory. This in turn means that a quantum field theory in the strong coupling limit can be formulated and the spectrum obtained.
\end{abstract}

It is common wisdom that Green function methods are typical of linear systems. There is no way to write down a solution to a non-homogeneous non-linear problem in a closed analytical form using the solution of the correspondent equation for the Green function.

The reason for this is quite easy to understand as the Green function method relies on an integral formula that implies the validity of the superposition principle. This point is particularly relevant in quantum field theory where strong coupling generally means to take the non-linear part of classical equations of the field theory without possibility to rely on the superposition principle and weak perturbation theory.

Recently we showed how a perturbation theory can be obtained for strongly perturbed field theories 1, , 2, 3, 44. The main result can be summed up as to have proved that a gradient expansion is a strong perturbation theory. This in turn implies that the leading order is generally a classical equation in the form

$$
\ddot{\phi}(t)+P(\phi(t))=0
$$

being $P(\phi(t))$ a generic non-linear function of the field $\phi(t)$. A Green function can anyway be defined in this case by looking for a solution of the equation

$$
\ddot{\phi}(t)+P(\phi(t))=A \delta(t)
$$

being $A$ a constant scale factor, but there is no way to attach a meaning to a solution of the equation

$$
\ddot{\phi}(t)+P(\phi(t))=j(t)
$$

being $j(t)$ a given source, in the well-known form

$$
\phi(t)=\int_{-\infty}^{+\infty} G\left(t-t^{\prime}\right) j\left(t^{\prime}\right) d t^{\prime}
$$

Anyhow, using a pure numerical approach, we are going to show that although eq.(4) is not exactly applicable, an approximate solution is given in this case by

$$
\phi(t) \approx \int_{0}^{t} G\left(t-t^{\prime}\right) j\left(t^{\prime}\right) .
$$

On a mathematical standpoint this appears as the leading order of an expansion to hold in the strong coupling limit, when we expand with respect to a given large parameter in eq.(1). Then, when we interpret the Green function in quantum field theory as containing all the spectrum, we are able to obtain it in the limit of a strong coupling limit holding at the leading order. Indeed we used this approach to obtain the spectrum in the strong coupling limit of a $\phi^{4}$ theory [3]. Here we will support our finding by applying this approach also to the pendulum equation

$$
\ddot{\vartheta}(t)+\sin (\vartheta(t))=0
$$

that appears to be the leading order of a gradient expansion of the sine-Gordon model in quantum field theory. We will obtain the spectrum for this field theory in the strong coupling limit, that is, when the non-linear term is taken to be largely increasing.

Firstly, let us review the situation of a $\phi^{4}$ case. A gradient expansion gives at the leading order the classical equation

$$
\ddot{\phi}(t)+\phi^{3}(t)=0 .
$$




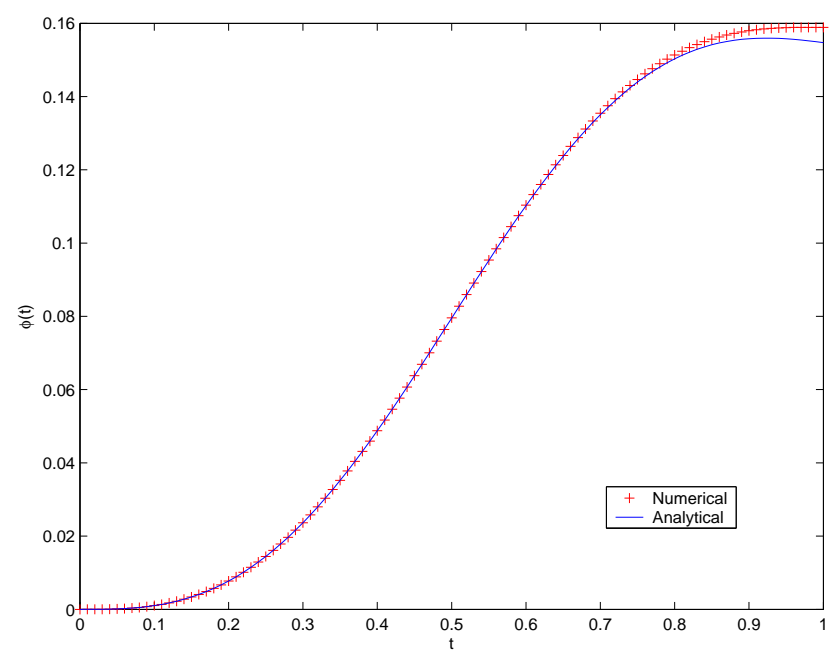

FIG. 1: Agreement between numerical and analytical solutions for $\phi^{4}$ case with a sine forcing.

We are interested to the numerical solution of the non-homogeneous equation

$$
\ddot{\phi}(t)+\phi^{3}=\sin (2 \pi t) .
$$

The solution of the Green function equation

$$
\ddot{G}(t)+G(t)^{3}=\delta(t)
$$

is

$$
G(t)=\theta(t) 2^{\frac{1}{4}} \mathrm{Sn}\left[\left(\frac{1}{2}\right)^{\frac{1}{4}} t, i\right]
$$

being sn the Jacobi snoidal function and $\theta(t)$ the Heaviside function. So, we compare our numerical solution with the analytical formula

$$
\phi(t) \approx 2^{\frac{1}{4}} \int_{0}^{t} \operatorname{sn}\left[\left(\frac{1}{2}\right)^{\frac{1}{4}}\left(t-t^{\prime}\right), i\right] \sin \left(2 \pi t^{\prime}\right) d t^{\prime} .
$$

The results are given in fig.(11). The agreement appears to be perfect till the end of the integration interval where both curves start to differ. This is a typical behavior of a term of a perturbation series. We have also tried to change the driving term. We have considered the case $j(t)=e^{-t}$ and the result is given in fig.(2). Again the agreement is practically perfect for a large part of the integration interval.

The Sine-Gordon equation has an exterminate literature. The quantum model in $1+1$ dimensions was firstly considered by Coleman [5] obtaining the classical result that this model is equivalent to the massive Thirring model. Through a gradient expansion at the leading order, a forced pendulum can be obtained from the Sine-Gordon equation written in the form

$$
\ddot{\vartheta}-\nabla^{2} \vartheta+\sin (\vartheta)=j
$$

A forced Sine-Gordon equation has been considered in [6, 7]. Our case applies when the non-linear term is very large. In this case one can show that a gradient expansion is obtained [1, 2, 3, 4]. We will give a proof of this below. So, we have to solve the forced pendulum equation

$$
\ddot{\vartheta}(t)+\sin (\vartheta(t))=j(t) .
$$

In order to apply our approach we consider the following Green function

$$
G(t)=2 \theta(t) \mathrm{am}\left[\frac{1}{\sqrt{2}} t, \sqrt{2}\right],
$$




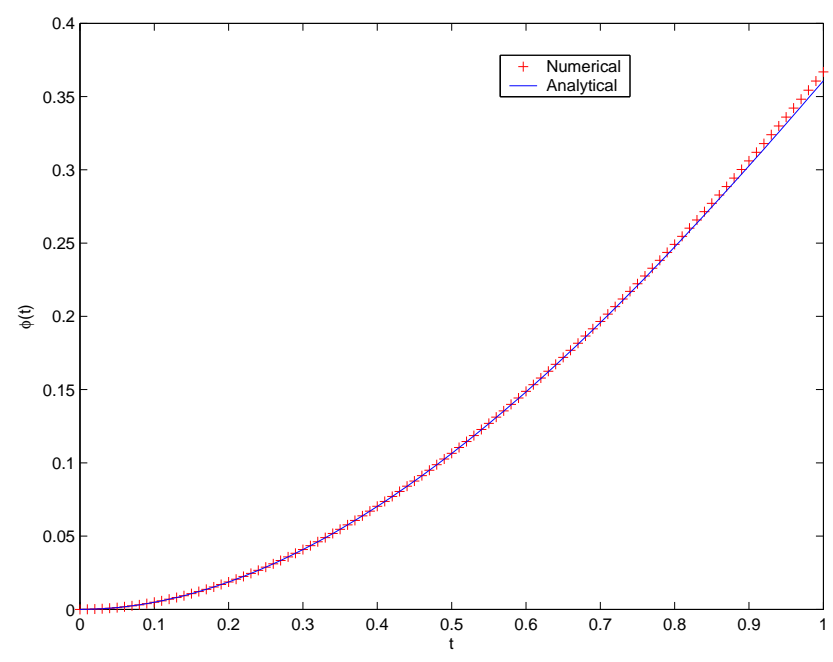

FIG. 2: Agreement between numerical and analytical solutions for $\phi^{4}$ case with an exponential forcing.

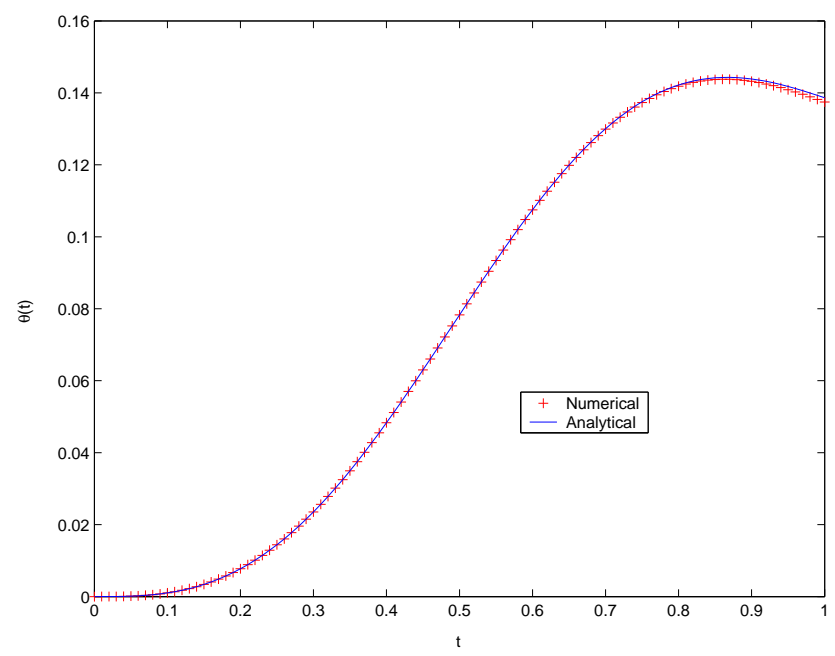

FIG. 3: Agreement between numerical and analytical solutions for the pendulum case with a sine forcing.

being am the Jacobi amplitude, that solves the equation

$$
\ddot{G}(t)+\sin (G(t))=\sqrt{2} \delta(t) .
$$

Then, with a forcing sine function we get

$$
\theta(t) \approx \frac{2}{\sqrt{2}} \int_{0}^{t} \operatorname{am}\left[\frac{1}{\sqrt{2}}\left(t-t^{\prime}\right), \sqrt{2}\right] \sin \left(2 \pi t^{\prime}\right) d t^{\prime} .
$$

having rescaled by $\sqrt{2}$ to take into account the definition eq.(15) As shown in Fig.(3), the agreement between numerical and analytical results is satisfactory.

The relevance of this result relies on the fact that is quite easy to write down a generating functional for a leading order quantum field theory. Indeed, the classical theory is very similar to a free linear theory. We have already used this fact in Ref. [3] to obtain the spectrum and the propagator of a $\lambda \phi^{4}$ theory in the limit of very large $\lambda$. We have shown that the generating functional can be written down as

$$
Z[j]=\exp \left[\frac{i}{2} \int d^{D} x d^{D} x^{\prime} j(x) \Delta\left(x-x^{\prime}\right) j\left(x^{\prime}\right)\right]
$$


being $\Delta\left(x-x^{\prime}\right)$ the Feynman propagator. In our case this propagator can be easily obtained using the fact that we have two solutions to eq.(9) and eq.(15) obtained just by the change $t \leftrightarrow-t$. In this way we are able to recover our solution eq.(5) as it should be with the proper time evolution. This can be easily accomplished when the Feynman propagator is written down as [3]

$$
\Delta(x)=-i \delta^{(D-1)}(x)[G(t)+G(-t)]
$$

to take into account the given time ordering. So, let us consider a source $j(t)$ starting at $\mathrm{t}=0$ at increasing time. One has

$$
\frac{\delta Z[j]}{\delta j(x)}=\phi(x)=\int_{0}^{t} \tilde{G}\left(t-t^{\prime}\right) j\left(t^{\prime}\right) d t^{\prime}
$$

being $\tilde{G}$ the Green function with the Heaviside function removed and we have integrated the Dirac distribution on the spatial coordinates.

In order to write down both the spectrum and the propagator for our case we use the following results [8]:

$$
\operatorname{sn}(u, i)=\frac{2 \pi}{K(i)} \sum_{n=0}^{\infty} \frac{(-1)^{n} e^{-\left(n+\frac{1}{2}\right) \pi}}{1+e^{-(2 n+1) \pi}} \sin \left[(2 n+1) \frac{\pi u}{2 K(i)}\right]
$$

being $K(i)=\int_{0}^{\frac{\pi}{2}} \frac{d \theta}{\sqrt{1+\sin ^{2} \theta}} \approx 1.3111028777$ a constant. Similarly,

$$
\operatorname{am}(u, \sqrt{2})=\frac{\pi u(1+i)}{2 K(i)}+\sum_{n=1}^{\infty} \frac{1}{n} \frac{(-i)^{n} e^{-n \frac{\pi}{2}}}{1+(-1)^{n} e^{-n \pi}} \sin \left[\frac{n \pi u(1+i)}{2 K(i)}\right] .
$$

Our aim is to get the time dependent part of the propagator in the form

$$
\tilde{G}(t)=\sum_{n=-\infty}^{\infty} A_{n} e^{-i E_{n} t}
$$

being $A_{n}$ constant amplitudes and $E_{n}$ the spectrum of the given quantum field theory. For the $\phi^{4}$ theory one has easily

$$
\tilde{G}(t)=\sum_{n=0}^{\infty} \frac{\pi}{i K(i)} \frac{(-1)^{n} e^{-\left(n+\frac{1}{2}\right) \pi}}{1+e^{-(2 n+1) \pi}}\left[e^{i(2 n+1) \frac{\pi}{2^{\frac{5}{4}} K(i)} t}-e^{-i(2 n+1) \frac{\pi}{2^{\frac{5}{4}} K(i)} t}\right]
$$

and we are able to read out the spectrum being given by that of a harmonic oscillator

$$
E_{n}=(2 n+1) \frac{\pi u}{2^{\frac{5}{4}} K(i)}
$$

for the particle content of the theory in the strong coupling limit [3]. Similarly one has

$$
A_{n}=\frac{\pi}{i K(i)} \frac{(-1)^{n} e^{-\left(n+\frac{1}{2}\right) \pi}}{1+e^{-(2 n+1) \pi}}
$$

and its complex conjugate. For the Sine-Gordon case we arrive at a rather unexpected result that the quantum theory in a strong coupling limit (increasingly large mass) has complex energy eigenvalues. Indeed, one has

$$
\tilde{G}(t)=\frac{\pi(1+i) t}{\sqrt{2} K(i)}+\sum_{n=1}^{\infty} \frac{1}{n} \frac{(-i)^{n} e^{-n \frac{\pi}{2}}}{1+(-1)^{n} e^{-n \pi}} \sin \left[\frac{n \pi(1+i) t}{2 \sqrt{2} K(i)}\right]
$$

and this gives the result

$$
\tilde{G}(t)=\frac{\pi(1+i) t}{\sqrt{2} K(i)}+\sum_{n=1}^{\infty} \frac{1}{i n} \frac{(-i)^{n} e^{-n \frac{\pi}{2}}}{1+(-1)^{n} e^{-n \pi}}\left[e^{i \frac{n \pi t}{2 \sqrt{2} K(i)}} e^{\frac{-n \pi t}{2 \sqrt{2} K(i)}}-e^{-i \frac{n \pi t}{2 \sqrt{2} K(i)}} e^{\frac{n \pi t}{2 \sqrt{2} K(i)}}\right]
$$


that means

$$
E_{n}=\frac{n \pi}{\sqrt{2} K(\sqrt{2})}
$$

being $K(\sqrt{2})=K(i)(1-i)$ and $n$ starting at $n=1$. We note also the presence of a zero mode. It should be noticed that the Green function of the Sine-Gordon model is bounded in the limit $t \rightarrow \infty$ making sense of complex eigenvalues, but each single mode is unstable with respect to time evolution. This in turn implies that physical results, obtained using the full Green function, are also finite. We note that this situation has been studied in Bose-Einstein condensates [9].

In order to complete our analysis, we show how a gradient expansion emerges as a strong coupling expansion for the particular case of the Sine-Gordon model. This analysis also applies to the $\phi^{4}$ model as already shown [1, 2, 3, 4]. We reintroduce the parameters into eq.(12) to write

$$
\ddot{\vartheta}-\nabla^{2} \vartheta+\frac{\alpha}{\beta} \sin (\beta \vartheta)=0
$$

being $\alpha$ a squared mass term and we take $\beta=1$ preserving consistency in agreement to the analysis given by Coleman [5]. A gradient expansion is recovered by rescaling time as $t \rightarrow \sqrt{\alpha} t$ and putting

$$
\vartheta=\vartheta_{0}+\frac{1}{\alpha} \vartheta_{1}+\frac{1}{\alpha^{2}} \vartheta_{2}+\ldots
$$

showing that we do have a strong coupling expansion that holds in the limit $\alpha \rightarrow \infty$. Indeed, we get the following non trivial set of equations

$$
\begin{aligned}
\ddot{\vartheta}_{0}+\sin \left(\vartheta_{0}\right) & =0 \\
\ddot{\vartheta}_{1}+\cos \left(\vartheta_{0}\right) \vartheta_{1} & =\nabla^{2} \vartheta_{0} \\
\ddot{\vartheta}_{2}+\cos \left(\vartheta_{0}\right) \vartheta_{2} & =\nabla^{2} \vartheta_{1}+\frac{1}{2} \sin \left(\vartheta_{0}\right) \vartheta_{1}^{2}
\end{aligned}
$$

Adding a source turns us back to eq. (13) for the leading order. This complete our proof.

Although we have shown, through numerical analysis, that for some nonlinear models the Green function appears to work, only an analytical proof could move our results from a conjectural state to a fully proved theorem. Notwithstanding this aspect of our approach, the results are really unexpected as it is a general wisdom to assume that only linear problems could be treated through Green function methods, as happens also to the leading order of a weak perturbation theory. The possibility to get a full spectrum of a quantum field theory, in some limit, is a very relevant by-product of this method and the nature of a gradient expansion, both in the classical and quantum limits, is elucidated appearing as a natural strong coupling expansion.

\footnotetext{
* marcofrasca@mclink.it

[1] M. Frasca, hep-th/0610148, to appear in International Journal of Modern Physics A.

[2] M. Frasca, Int. J. Mod. Phys. D 15, 1373 (2006).

[3] M. Frasca, Phys. Rev. D 73, 027701 (2006); Erratum-ibid. D 73, 049902 (2006).

[4] M. Frasca, hep-th/0509125, to appear in International Journal of Modern Physics A.

[5] S. Coleman, Phys. Rev. D 11, 2088 (1975).

[6] G. Fiore, math-ph/0512002

[7] A. D'Anna, M. De Angelis, G. Fiore, math-ph/0507005.

[8] I. S. Gradshteyn, I. M. Ryzhik, Table of Integrals, Series, and Products, (Academic Press, 2000).

[9] M. Mine, M. Okumura, T. Sunaga, Y. Yamanaka, cond-mat/0609052.
} 